\title{
Cost effective strategy using Kriging surrogates to compute fatigue at multiple locations of a structure: Application to offshore wind turbine certification
}

\author{
Quentin Huchet ${ }^{1,2, *}$, Cécile Mattrand ${ }^{2}$, Pierre Beaurepaire ${ }^{2}$, Nicolas Relun ${ }^{1}$ and Nicolas Gayton ${ }^{2}$ \\ ${ }^{1}$ EDF R\&D, Dpt ERMES, 7 Boulevard Gaspard Monge, 91120 Palaiseau, France \\ ${ }^{2}$ Université Clermont Auvergne, CNRS, SIGMA Clermont, Institut Pascal, F-63000 Clermont-Ferrand, France
}

\begin{abstract}
Offshore wind energy development has experienced a rapid development over the last few years encouraged by the of carbon reduction policy and the energy mix objectives of several countries worldwide. Nowadays, the offshore projects under investigation are composed of tens to hundreds of units reaching impressive dimensions with total rotor diameters from 150 to 220 meters and production capacity of 5 to $12 \mathrm{MW}$ per turbine. Mechanical analyses have to be performed to validate the design regarding the solicitations it may face during its lifetime (20 years). Because of the high number of solicitation cycles the structure is confronted to, an estimation of the cumulated damage is mandatory and has to be carefully assessed. As presented in standards, this verification requires massive computation investments and is usually a challenging task for project engineers. This paper presents the "MultiSite" extension of the AK-DA numerical strategy ("Adaptive Kriging for Damage Assessment"). After being formalized, an illustration of its behaviour and performances is proposed for the validation of a design regarding its cumulated damages at different locations.
\end{abstract}

\section{Introduction}

The reduction of carbon cost is a one the most important challenge in today's energy production industry. Wind energy is one the most developing technology with an increase of more than $10 \%$ per year [1]. To ensure the sustainable development of this technology, the reduction of the production cost is required. To that end, the offshore wind production is being increasingly developed because of the greater wind conditions and the shore distance which permits to elevate larger structures with a reduced risk of collapse on people.

To guarantee the project development and secure investments, each of the structural designs has to be certified regarding its mechanical behaviour. In this context, certification bodies propose full-defined analysis frameworks [2] based on standards [3,4] and listing all the numerical assessments engineers have to perform in order to validate the design or not. Because of the high number of cycles the structures undergo during their lifetime, mechanical fatigue analyses have to be carefully assessed. The total lifetime of the structure is considered as a series of elementary "Conceptual Situations" (CS), some of which requiring an estimation of the cumulated damage. An environmental model is then defined and parameterized with in-situ observable quantities such as the 10-min wind mean speed or the significant wave-height. The entire solicitation domain is represented as a regular grid of parameter combinations. The size of this grid depends on the number of environmental parameters and the chosen discretization intervals. As an example, the estimation of the cumulated damage during the "Normal production" situation (DLC 1.2 in [3]) involves up to hundreds of thousands combinations of environmental parameters.

For each of the above-defined combinations of parameters, engineers have to perform a fatigue analysis to quantify their potential short-term damage impact on the structure. The computation of this damage, noted $d$ in this paper, is a demanding task. A combination of parameters is firstly used to generate random trajectories of environmental solicitations for the wind and the sea state. Secondly, the structural responses are time computed with multiphysics codes dealing with aerodynamics, hydrodynamics, structural mechanics and turbine servo adaptations. These responses are finally post-treated in order to extract the stress responses and a Rainflow cycle counting is operated for different locations of the analysed design. For each of the location of interest, a short-term damage $d$ is computed by the use of Miner's cumulative law as:

$$
d(\boldsymbol{x}, \omega)=\sum_{k=1}^{n_{R F}(\omega)} \frac{1}{N_{k}(\boldsymbol{x}, \omega)}
$$

with $\boldsymbol{x}=\left[x_{1}, \ldots, x_{n}\right]^{T}$ the vector of the $n$ environmental parameters, $n_{R F}$ the number of cycles extracted by the Rainflow counting and $N_{k}$, the number of cycles to failure with a given stress. $\omega$ stands for the random

Corresponding author: quentin.huchet $@$ sigma-clermont.fr 
character of the solicitations for a given combination of environmental solicitation. In this paper, the random seed is fixed for the generation of solicitation time series, therefore discarding this variability. Considering this numerical chain, the simulation time is usually larger than the simulated solicitation period, leading to demanding analyses.

A mean damage of the cycle conceptual situation is estimated as a weighted sum of short-term damages as:

$$
D=\sum_{k=1}^{n_{c}} d\left(\boldsymbol{x}_{k}\right) p_{k} \Delta
$$

with $p_{k}$ defined as the probability of occurrence of the combination of environmental parameters $\boldsymbol{x}_{k}$ ("metocean" data collected in-situ) and $\Delta$, the discretization unit provided by the certification guidelines.

Considering $n_{s}$ sites (structural locations of interest), the design is validated regarding its resistance to mechanical damage if:

$$
D_{i}<D_{i}^{\text {lim }} \text { for all } i \in\left[1, \ldots, n_{s}\right]
$$

with $D_{i}^{\text {lim }}$ the damage limit threshold for the location $i$.

In order to reduce the simulation costs related to the estimation of the global mean damage $D$, the AK-DA ("Adaptive Kriging for Damage Assessment") algorithm has been proposed in [5]. Based on adaptive Kriging predictions of the short-term model responses $d$, it aims at iteratively constructing a reduced but informative design of experiments (set of environmental parameter combinations) to accurately approximate the demanding quantity $D$ for a single location of the structure. Although being computationally efficient, only one location at a time could be investigated which reduces its potential industrial applicability. This paper presents an extension of this method named AK-DA MultiSite and developed to tackle this shortcoming, i.e. to adress simultaneously several locations. Firstly, the section 2 focuses on a brief explanation of the AK-DA algorithm in its initial formalization (single location approach). Section 3 presents the "MultiSite" formalization, which is illustrated in section 4. Conclusions and future developments are presented in section 5 .

\section{Estimation of the damage using the AK-DA method (Single location approach)}

The AK-DA method was proposed to reduce the simulation costs for the estimation of the global mean damage $D$, presented in Eq. (2).

\subsection{The Kriging Prediction}

Kriging is a statistical method aiming at predicting a phenomenon response based on a limited set of realizations (model observations). Its development in the last decades made it widely spread in numerical analysis
[6]. Let $m$ be a numerical model mapping the input $\boldsymbol{x}=\left[x_{1}, \ldots, x_{n}\right]^{T} \in \mathcal{D}_{\boldsymbol{x}}$ to a scalar output $y \in \mathcal{D}_{y}$. This model is assumed to be a "black-box" function from which a set of $n_{\text {obs }}$ observations $\left\{\mathbf{X}_{o b s}, \boldsymbol{y}_{\text {obs }}\right\}$ is available (model runs). Kriging assumes the model response $y_{0}$ for a non-observed input $\boldsymbol{x}_{0} \in \mathcal{D}_{\boldsymbol{x}}$ is the realization of a Gaussian process presented as:

$$
y_{0}=\sum_{i}^{p} f_{i}\left(\boldsymbol{x}_{0}\right) \beta_{i}+z\left(\boldsymbol{x}_{0}\right)=\boldsymbol{f}_{0}^{\mathrm{T}} \boldsymbol{\beta}+z_{0}
$$

This formalization aggregates two contributions. A regressive term is firstly used to capture the global trend of the model response based on the observational set. This part is based on a $p$-order regression of basis vector $\boldsymbol{f}$. To calibrate the vector of weights $\boldsymbol{\beta}$, statistical analyses are to be performed such as generalized least square estimations. The best estimation of this coefficient vector regarding the observational set is hereafter noted $\widehat{\boldsymbol{\beta}}$. This deterministic part is completed with a stochastic contribution, noted $z_{0}$, and assumed to be a realization of a Gaussian process $z$ of zero-mean $(\mathbb{E}[z]=0)$. The covariance kernel of this structure is presented as:

$$
\operatorname{COV}\left(z_{1}, z_{2}\right)=\sigma_{z}^{2} r\left(\boldsymbol{x}_{1}, \boldsymbol{x}_{2}, \boldsymbol{\theta}\right)
$$

with $\sigma_{z}^{2}$ the process variance, $r$ the correlation model of parameter vector $\boldsymbol{\theta}$ and $\boldsymbol{x}_{1}, \boldsymbol{x}_{2} \in \mathcal{D}_{\boldsymbol{x}}$. This contribution presents the advantage of introducing local adjustments based on a spatial dependence structure between model responses. For accuracy purposes, the calibration of the vector of hyperparameters $\boldsymbol{\theta}$ is crucial. Its best estimator $\widehat{\boldsymbol{\theta}}$ is obtained from a statistical analysis of the observations (e.g. maximum likelihood estimations, cross validation methods).

Within this formalization, model responses for nonsimulated points can be statistically predicted. The "Best Linear Unbiased Predictor" (BLUP) is well suited for numerical science applications. For all $\boldsymbol{x}_{0} \in \mathcal{D}_{x}$, it proposes to compute a predictive density function $\mathcal{N}\left(\hat{y}_{0}, \sigma_{0}\right)$ of the real response $y_{0}$ with parameters defined as:

$$
\hat{y}_{0}=\boldsymbol{f}_{0}^{\mathrm{T}} \widehat{\boldsymbol{\beta}}+\boldsymbol{r}_{0}^{\mathrm{T}} \mathbf{R}^{-\mathbf{1}}\left(y_{o b s}-\mathbf{F} \widehat{\boldsymbol{\beta}}\right)
$$

$$
\begin{aligned}
\sigma_{0}^{2}=\sigma_{z}^{2}\left[1-\boldsymbol{r}_{0}^{\mathrm{T}}\right. & \mathbf{R}^{-\mathbf{1}} \boldsymbol{r}_{0} \\
& \left.+\boldsymbol{u}_{0}^{\mathrm{T}}\left(\mathbf{F}^{\mathrm{T}} \mathbf{R}^{-1} \mathbf{F}\right)^{-\mathbf{1}} \boldsymbol{u}_{0}\right]
\end{aligned}
$$

with $\boldsymbol{u}_{0}=\mathbf{F}^{\mathrm{T}} \mathbf{R}^{-1} \boldsymbol{r}_{0}-\boldsymbol{f}_{0}, \quad \mathbf{F} \quad$ and $\boldsymbol{f}_{0} \quad$ respectively denoting the matrix of regression evaluated for the observation points and the vector of regression evaluated for the prediction input $\boldsymbol{x}_{0} . \mathbf{R}$ and $\boldsymbol{r}_{0}$ respectively denote the matrix of correlation evaluated for the observation set and the vector of cross correlation between the input $\boldsymbol{x}_{0}$ and the observations.

For practical purposes, model predictions are preferred to be deterministic and are thus set to the most 
likely value, namely the distribution mean: $y_{0} \approx \hat{y}_{0}$. An interesting feature of the Kriging prediction is the interpolating character of the model approximation $\left(y_{0}=\hat{y}_{0}\right.$ if $\boldsymbol{x}_{0}$ is contained in the inputs $\left.\mathbf{X}_{o b s}\right)$. The prediction variance $\sigma_{0}^{2}$ gives information on the accuracy of the prediction, evaluating its statistical error.

Additional explanations, demonstrations and proofs are available in [7].

\subsection{The AK-DA method}

As introduced in Section 1, the estimation of cycle damages presented in Eq. (2) can be demanding during the design stage of the wind turbine structures. The idea of the AK-DA algorithm is to reduce the simulation costs by reducing the number of short-term damage estimations based on the real code. From this reduced set of observations, a Kriging surrogate model $\hat{d}$ of the demanding response $d$ is computed so as the related statistical estimation of the prediction error $\sigma_{d}^{2}$ for the complete grid of environmental combinations from the standards. An estimator $\widehat{D}$ of the cumulative damage $D$ is computable by replacing the real model response $d$ by its computed surrogate model $\hat{d}$ :

$$
\widehat{D}=\sum_{j=1}^{n_{c}} \hat{d}\left(\boldsymbol{x}_{j}\right) p_{j} \Delta
$$

The variance $\sigma_{\widehat{D}}^{2}$ of the estimator therefore reads:

$\sigma_{\widehat{D}}^{2}=\sum_{j=1}^{n_{c}} \sum_{k=1}^{n_{c}} p_{j} p_{k} \operatorname{COV}\left(\hat{d}\left(\boldsymbol{x}_{j}\right), \hat{d}\left(\boldsymbol{x}_{k}\right), \widehat{\boldsymbol{\theta}}\right) \Delta^{2}$

The quality of this approximation is highly influenced by the model observations. AK-DA proposes an iterative enrichment of an initial set of observation to enhance the accuracy of the estimator $\widehat{D}$.

Figure 1 illustrates the construction of the design of experiments. Considering the iter $^{\text {th }}$ enrichment step, the design of experiments $\left\{\mathbf{X}_{\text {iter }}, \boldsymbol{d}_{\text {iter }}\right\}$ is used to calibrate a Kriging surrogate model $\hat{d}_{i t e r}$ for the prediction of the damage model responses $d$ for all the non-simulated environmental combinations of parameters. An estimation of the quantity of interest $\widehat{D}_{i t e r}$ is computed from Eq. 8 as the related coefficient of variation $\delta_{i t e r}$ :

$$
\delta_{i t e r}=\frac{\sigma_{\widehat{D}_{i t e r}}}{\widehat{D}_{\text {iter }}}
$$

While the stopping criterion is not verified $\left(\delta_{i t e r} \geq\right.$ $\delta_{t}$ ), the design of experiments is enriched. To do so, the variance $\sigma_{\tilde{D}_{i t e r}}^{2}$ of the global damage predictor (Eq. 9) is decomposed as:

$$
\sigma_{\tilde{D}_{i t e r}}^{2}=\sum_{j=1}^{n_{c}} \mathcal{C}_{j}
$$

Each of the contributions $\mathcal{C}_{j}\left(j \in\left[1, \ldots, n_{c}\right]\right)$ represents the part of a specific environmental combination of parameters $\boldsymbol{x}$ on the uncertainty of the estimator $\widehat{D}_{i t e r}$. This contribution is presented as:

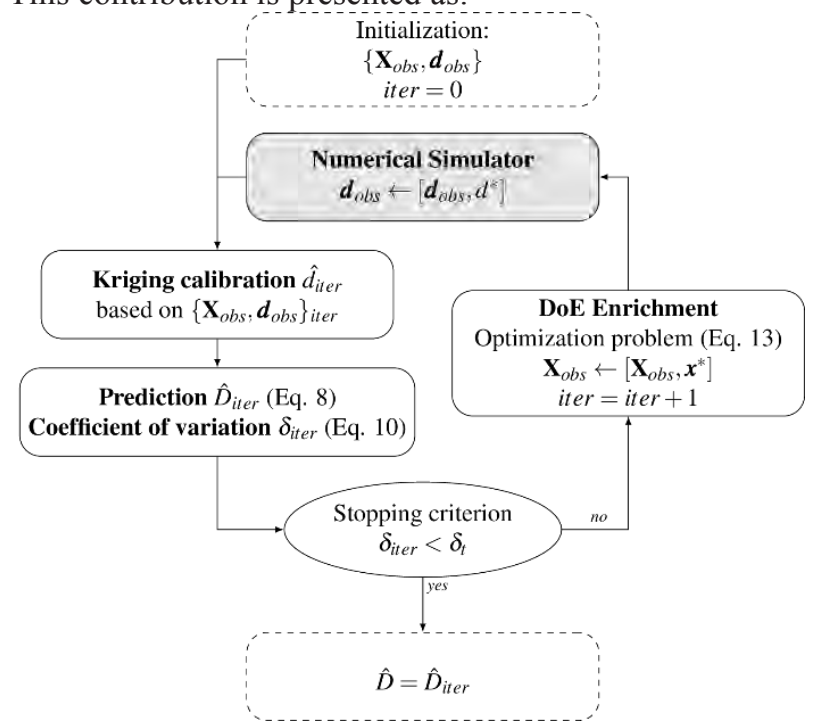

Fig. 1. AK-DA algorithm scheme

$$
\mathcal{C}_{j}=p_{i} \sum_{k=1}^{n_{c}} p_{k} \operatorname{COV}\left(\hat{d}\left(\boldsymbol{x}_{i}\right), \hat{d}\left(\boldsymbol{x}_{k}\right), \widehat{\boldsymbol{\theta}}\right) \Delta^{2}
$$

The objective is to reduce $\sigma_{\bar{D}}^{2}$. Hence, the selection of the environmental combination of parameters $\boldsymbol{x}^{*}=\boldsymbol{x}_{i^{*}}$ to enrich the design of experiments with is carried out by solving:

$$
j^{*}=\underset{j \in \bar{S}}{\operatorname{argmax}} \mathcal{C}_{j}
$$

with $\bar{S}$ regrouping the indices of the non-simulated environmental parameters.

A simulator run is then performed to observe the model response $d^{*}$ for the elected combination of environmental parameters $\boldsymbol{x}^{*}$ and the observation set is updated. Sequentially, the design of experiments is enriched and the prediction $\widehat{D}_{\text {iter }}$ gets more accurate.

Once the convergence criterion is reached $\left(\delta_{\text {iter }}<\right.$ $\delta_{t}$ ), an estimator $\widehat{D}$ of the demanding quantity of interest $D$ and based on an observation set $\left\{\mathbf{X}_{o b s}, \boldsymbol{d}_{o b s}\right\}$. The uncertainty of this estimator has to be taken into account in order to accept or to reject the proposed design. For such purpose, the deterministic acceptation criterion presented in Eq. (3) is transformed to consider the uncertainty regarding the estimator $\widehat{D}$ failure probability $P_{f}$. The validation criterion is presented as:

$$
P_{f}=P\left(D>D^{l i m} \mid\left\{\mathbf{X}_{o b s}, \boldsymbol{d}_{o b s}\right\}\right)<P_{\text {admit }}
$$

$P_{\text {admit }}$ is the probability threshold not to overpass for the design to be accepted.

Although being computationally efficient with accurate estimations and important reductions of the efforts [5], this method is not well-suited for industrial 
uses in this first formulation. During the design stage of wind turbine structures, several locations of the structures (sites) are to be analysed. For each, an estimation of the long-term damage quantities is to be assessed. The current method does not allow to perform this simultaneous analysis. In the following, an extension of the algorithm is presented to perform damage analyses for multiple locations of a structure in a single process.

\section{The AK-DA MultiSite extension}

The MultiSite extension of the AK-DA algorithm aims at adapting the approximation method to the analysis of multiple structural sites at the same time. As the single location version, this work proposes to efficiently approximate $n_{s}$ global damage quantities of interest, namely $D_{i}$ with $i \epsilon\left[1, \ldots, n_{s}\right]$. For each of the analysed sites, the grid of environmental parameters to simulate is unchanged and a single simulator run outputs $n_{s}$ short term damages, regrouped in an output vector $\boldsymbol{d}=$ $\left[d_{1}, \ldots, d_{n_{S}}\right]^{T}$. In this sense, the design of experiments is the same for each of the $n_{s}$ analyses. From the discretization of the environmental parameters, $n_{s}$ quantities of interest and their predictions are computed as:

$$
\begin{aligned}
& D_{i}=\sum_{j=1}^{n_{c}} d_{i}\left(\boldsymbol{x}_{j}\right) p_{j} \Delta \quad \text { for } i \in\left[1, \ldots, n_{s}\right] \\
& \widehat{D}_{i}=\sum_{j=1}^{n_{c}} \hat{d}_{i}\left(\boldsymbol{x}_{j}\right) p_{j} \Delta \quad \text { for } i \in\left[1, \ldots, n_{s}\right]
\end{aligned}
$$

As depicted in Figure 2, the enrichment step is here modified in order to optimize the construction of the design of experiments. The idea is to reduce the simulator calls with respect to a one site to one site approach. The procedure is performed by a two-step strategy. Firstly, a "pilot location" is elected between each of the $n_{s}$ analysed sites considering its importance regarding the design validation and the uncertainty of the related estimator. This selection is proposed as:

$$
i^{*}=\underset{i / \delta_{i}>\delta_{t}}{\operatorname{argmax}} P\left(D_{i}>D_{i}^{l i m} \mid\left\{\mathbf{X}_{o b s}, \boldsymbol{d}_{i, o b s}\right\}\right)
$$

This criterion aims at focusing on risky locations regarding cycle damage response, organizing the enrichments to reduce the related estimation uncertainties. The pilot location is then used to proceed the AK-DA enrichment strategy as defined in Section 2. This iterative enlargement of the observation set occurs until the uncertainty regarding each of the $n_{s}$ analysed sites is sufficiently reduced. This is controlled by the evolution of their coefficients of variation $\delta_{i}$ and the stopping criterion is presented as:

$$
\delta_{i}>\delta_{t} \text { for } i \in\left[1, \ldots, n_{s}\right]
$$

To accept or to reject the proposed design, engineers could take into account the uncertainties regarding each of the $n_{s}$ estimators $\widehat{D}_{i}$. To do so, the method focuses on the related failure events defined as:

$$
\mathcal{E}_{i}=\left\{D_{i}>D_{i}^{\text {lim }}\right\} \text { for } i \in\left[1, \ldots, n_{s}\right]
$$

A design is accepted if each of the defined events are verified. This validation criteria is presented as:

$$
P_{f}=P\left(\bigcup_{i=1}^{n_{s}} \varepsilon_{i}\right)<P_{a d m i t}
$$

For practical purpose, the independence hypothesis of the failure events is assumed and the $P_{f}$ is approximated by the following upper bound:

$$
\sum_{i=1}^{n_{c}} P\left(\varepsilon_{i}\right)<P_{a d m i t}
$$

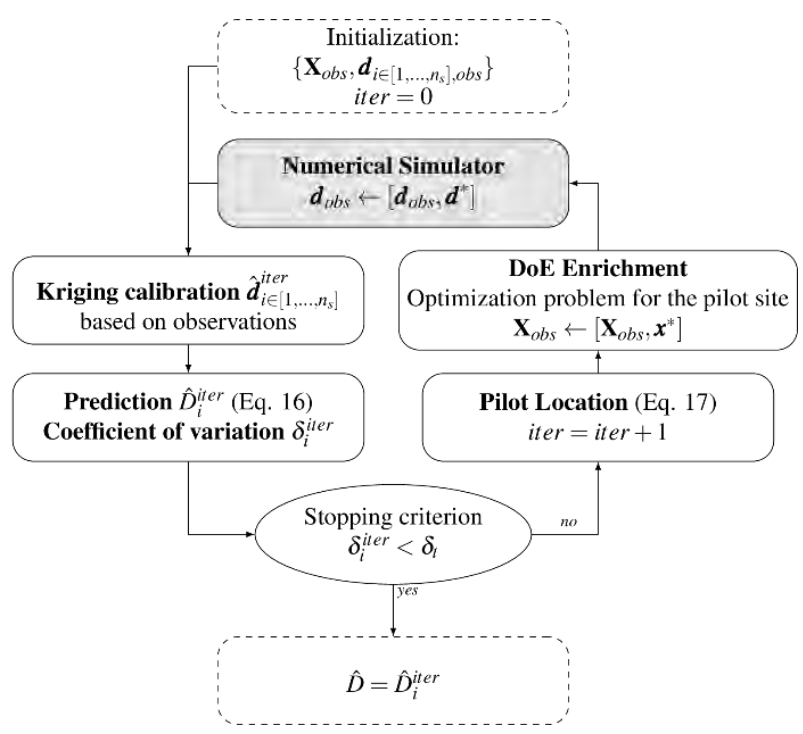

Fig. 2. AK-DA MultiSite algorithm scheme

\section{Numerical Illustration}

In this section, an industrial case is depicted to illustrate the behaviour of the proposed algorithm. The example, depicted in Figure 3, is a damage estimation of the DLC 1.2 [3] for the NREL OC3 Monopile [8]. Two parameters are set variable: the wind mean speed at hub height $(U)$ and the wind-wave misalignment $(\theta)$. The related metocean (probability of occurrence) is extracted from the generic data based proposed in [9].

The discretization of the parameter domain of variation is presented in Table 1 . The reference complete design of experiments is composed of $n_{c}=276$ combinations of environmental parameters. 
The analysis focuses on the four structural locations, presented in Figure 3. Four locations of interest are presented, composed of two points at the mudline and two points at the transition level (azimuts 0 and $90^{\circ}$ for each levels). For validation purpose, the complete discretization has been used to obtain the real model responses $d_{i}$ so as the reference values of the cycle mean damages $D_{i}(i \in[1, \ldots, 4])$. The algorithm is initialised with a reduced grid composed of 20 environmental combinations based on 4 wind speeds $(3,11,20,25 \mathrm{~m} / \mathrm{s})$ and 5 misalignments $\left(-150,-90,0,90,180^{\circ}\right)$. The Kriging predictions are all set with a constant basis of regression and the anisotropic "squared exponential" correlation model. The convergence criterion is set to $\delta_{t}=5 \%$ and the acceptance value is set to $P_{\text {admit }}=$ $10^{-4}$ for the damage limit $D^{\text {lim }}=10^{-5}$. These values are arbitrarily chosen for an illustration purpose

Table 1. Parameters of the study and discretization.

\begin{tabular}{|c|c|c|c|}
\hline Param. & $\begin{array}{c}\text { Domain of } \\
\text { definition }\end{array}$ & $\begin{array}{c}\text { Discretization } \\
\text { step }\end{array}$ & $\begin{array}{c}\text { Number of } \\
\text { Cases }\end{array}$ \\
\hline$U$ & 3 to $25 \mathrm{~m} / \mathrm{s}$ & $1 \mathrm{~m} / \mathrm{s}$ & 23 \\
\hline$\theta$ & {$[-150,360]^{\circ}$} & $30^{\circ}$ & 12 \\
\hline \multicolumn{3}{|c|}{ Number of environmental combinations } & 276 \\
\hline
\end{tabular}

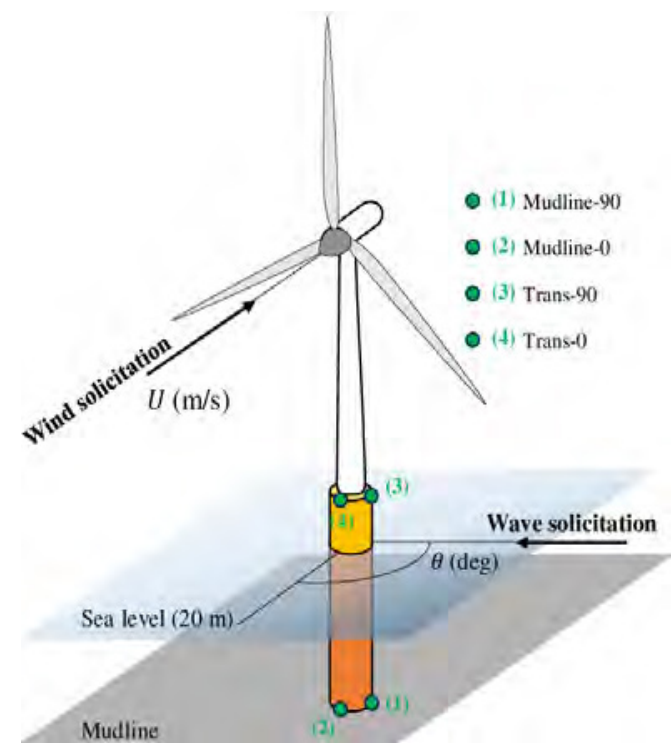

Fig. 3. NREL 5 MW Monopile with 2 environmental parameters and 4 locations of interest.

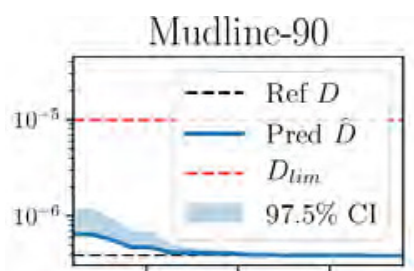

Trans-90

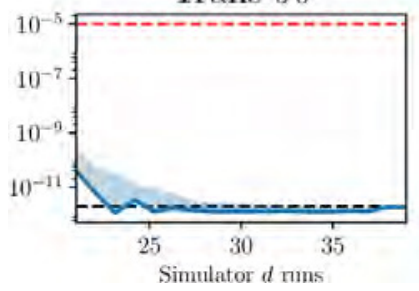

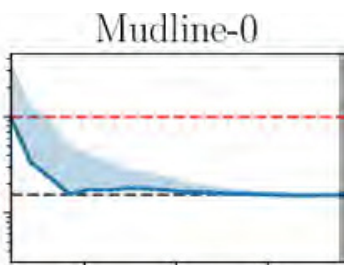

Trans- 0

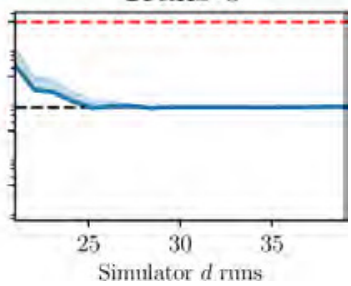

Fig. 4. Convergence of the damage estimators for each of the 4 analysed locations.

Figure 4 shows the evolution of the estimations $\widehat{D}_{i}$ of the cycle mean damages $D_{i}$ for the four studied locations of the structure. Firstly, it is to be noted that each of these values converge to the references within a reduced number of simulator calls (39 in total composed of 20 initial calls and 19 enrichments). The statistical errors of the estimations are depicted by the related $97.5 \%$ confidence intervals. The uncertainty progressively decreases in function of the iteration because of the information inflow of the model responses (enrichment of the observational set).

Table 2 regroups the results of estimations for the four locations of interest. At convergence, the four estimators perform with different accuracies. The most important locations regarding the damage validation are the mudline points. These two are well approximated with a maximum relative error (regarding the reference value) of $-1.4 \%$ for the $0^{\circ}$-azimuth location (Mudline- 0 ). The transition locations (Trans- 0 and Trans-90) show a lower cumulated damage far below the admitted limit value $D^{\text {lim }}=10^{-5}$. At convergence, the approximations of the damages are not as performant as the Mudline locations, with relative error of $-8.9 \%$ and $4.3 \%$ regarding the reference values.

Table 1. Estimations of the global damage estimated by the AK-DA MultiSite.

\begin{tabular}{|c|c|c|}
\hline & Mudline-90 & Mudline-0 \\
\hline Reference $D$ & $3.886 \times 10^{-7}$ & $1.548 \times 10^{-7}$ \\
\hline AK-DA $\widehat{D}$ & $3.876 \times 10^{-7}$ & $1.526 \times 10^{-7}$ \\
\hline Rel. error (\%) & -0.4 & -1.4 \\
\hline & Trans-90 & Trans-0 \\
\hline Reference $D$ & $2.029 \times 10^{-12}$ & $7.362 \times 10^{-12}$ \\
\hline AK-DA $\widehat{D}$ & $1.849 \times 10^{-12}$ & $7.681 \times 10^{-12}$ \\
\hline Rel. error (\%) & -8.9 & 4.3 \\
\hline
\end{tabular}

Figure 5 shows the evolution of the "pilot locations" elected by the algorithm at each iterations. This step shows a performant and economical behaviour with enrichments mainly controlled by the sizing locations (Mudline-0 and 90).

In total 39 simulator calls have been performed for the algorithm to converge. These observations are 
composed of 20 simulations from the initial reduced grid and 19 enrichments control by the AK-DA method. Comparing to the reference case based on a complete grid of size 276, the numerical gain in simulator calls is equal to 7.08 .

To validate or to discard the analysed design, the probability of failure $P_{f}$ is computed (Eq. 20 and 21). Fig. 6 depicts the evolution of this quantity for the 19 enrichments of the algorithm. The uncertainty related to the estimators, especially to the Mudline-0 location, makes the decision quantity high for the initial state ( $P_{f}>0.1$ for 21 model observations). The AK-DA enrichment strategy aims at reducing this uncertainty adding informative environmental situations to the observational set. The consequence is a rapid decreasing of the probability of failure reaching $P_{f}<10^{-11}$ after 5 iterations. From the $6^{\text {th }}$ enrichment, the probability of failure is considered as negligible. For this example, the design is validated regarding the cycle cumulated damages of the four structural locations.

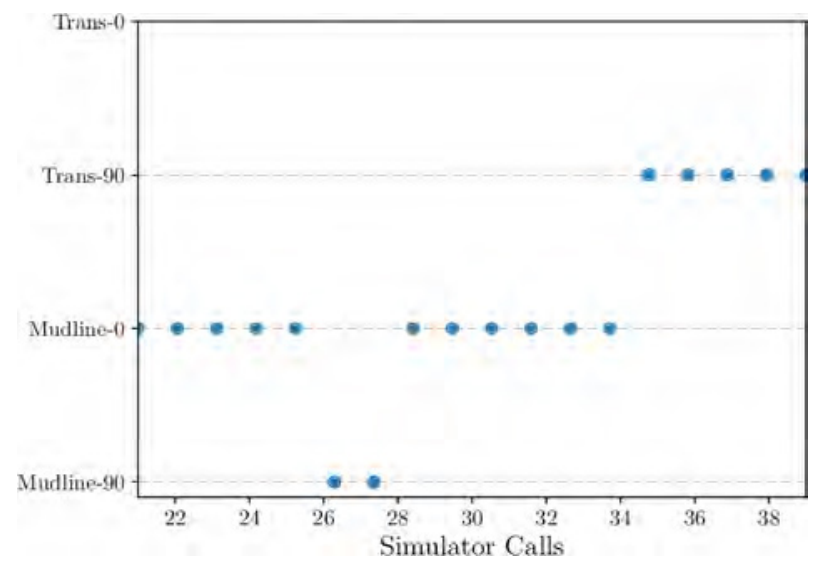

Fig. 5. Evolution of the pilot location for each of the AK-DA enrichments.

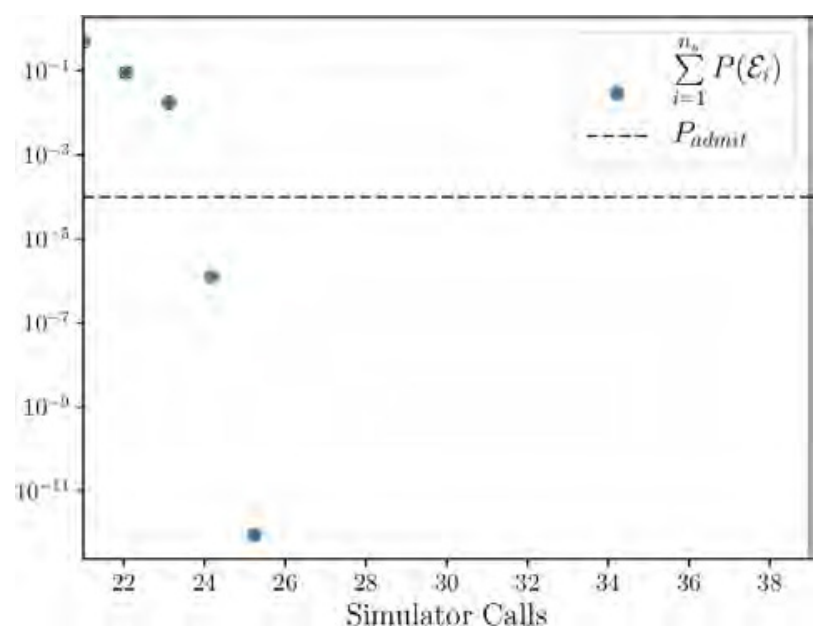

Fig. 6. Evolution of the admission criteria $P_{f}$ for each of the AK-DA enrichments.

\section{Conclusions and perspectives}

This paper presents an extension of the AK-DA (Adaptive Kriging for Damage Assessment) algorithm for the complete damage analysis of a design. The proposed MultiSite formalization aims at building a reduced but informative design of experiments to efficiently approximate the demanding cumulated damage quantities of a given structural design.

Based on parallel Kriging estimations, this algorithm is engineered in order to orientate the simulations for the most sizing locations to be accurately approximated with a prioritization of the enrichments regarding the uncertainty of the estimators and the risk of overpassing admitted damage limits. The validation criterion is here modified to take into consideration each of the possible failure events (damage threshold passing) in the decision step of the design validation.

The numerical example, based on an industrial case, illustrates the algorithm behaviour and the prioritization of the enrichments. The results are satisfying with an important reduction of the simulator calls (gain of 7) and effective approximations of the damage quantities.

Future works will focus on the use of this algorithm to perform statistical analyses of the structural damages that require a large number of estimations, i.e. uncertainty propagation, sensitivity analysis and reliability analysis.

This $\mathrm{PhD}$ project is financially supported by a CIFRE grand industrially supported by EDF (Palaiseau, France) and partially subsidized by the ANRT under grant \#2015/0692.

\section{References}

1. Global Wind Energy Council, Global wind report 2016. (2016)

2. DNV-GL, DNV-OS-J101 Design Of Offshore Wind Turbine Structures (2014)

3. IEC, 61400-1 Wind Turbines - Part 1; Design requirements (2005)

4. IEC, 61400-3 Wind Turbines - Part 3; Design requirements for offshore wind turbines (2009)

5. Q. Huchet, C. Mattrand, P. Beaurepaire, N. Relun, N. Gayton, A Kriging based procedure for the certification of wind turbine structures: application to large scale models. Procedings of the 54th ESReDA Seminar (2018)

6. T. W. Simpson, J. D. Poplinski, P. N. Koch, J. K. Allen, Metamodels for computer based engineering design : survey and recommendations. Engineering with computers 17-2 (2001)

7. V. Dubourg, Adaptive surrogate models for reliability analysis and reliability-based design optimization. PhD Thesis, Université Blaise Pascal Clermont-Ferrand II (2011)

8. J. Jonkman, S. Butterfield, W. Musial, G. Scott, Definition of a 5-MW reference wind turbine for offshore system development. National Renewable Energy Laboratory (NREL) (2009) 
9. G. M. Stewart, A. Robertson, J. Jonkman, A. Lackner. The creation of a comprehensive metocean data set for offshore wind turbine simulations. Journal of Wind Energy, 19-6 (2015) 